\title{
UTILIZAÇÃO DA FERRAMENTA PIXEL DO FACEBOOK PARA OTIMIZAÇÃO DE CAMPANHAS DE CONVERSÃO EM ANÚNCIO PAGO
}

\author{
UTILIZATION OF THE FACEBOOK PIXEL TOOL TO OPTIMIZE \\ CONVERSION OF CAMPAIGNS IN PAID ADVERTISING
}

\author{
Vítor Leal Vicentin - vitorlvicentin@gmail.com \\ Erick Eduardo Petrucelli - erick.petrucelli@fatectq.edu.br
}

Faculdade de Tecnologia de Taquaritinga (FATEC) -SP -Brasil

DOI: 10.31510/infa.v16i2.648

\begin{abstract}
RESUMO
No contexto do presente trabalho, Pixel trata-se de uma ferramenta da plataforma de mídia social Facebook, constituindo-se de um pequeno código JavaScript, podendo ser utilizada para fazer a marcação de usuários e, também, o rastreamento de suas ações dentro de um determinado site, assim trazendo dados estatísticos sobre o público e suas interações. Campanha de conversão é um modelo de campanha de marketing digital com o objetivo de aumentar as conversões, ou seja, garantindo que o usuário efetivamente faça uma ação previamente desejada, podendo ser de um simples cadastro em um site até uma compra de um determinado produto. Este artigo se inicia abordando a instalação do Pixel do Facebook em um site e demonstrando de forma teórica o seu potencial para auxiliar campanhas de marketing digital. Por fim, aborda um exemplo real da utilização do Pixel em uma campanha de conversão em anúncio pago no Facebook e discute os resultados alcançados.
\end{abstract}

Palavras-chave: Facebook. Pixel. Marketing Digital. Campanha de Conversão.

\begin{abstract}
In the context of this work, Pixel is a tool of the social media platform Facebook, consisting of a small JavaScript code that can be used to tag users and to track their actions within a given site, thus bringing statistical data about the audience and their interactions. Conversion campaign is a digital marketing campaign model that aims to increase conversions, that is, ensuring that the user effectively does a previously desired action, from a simple signup on a website to a purchase of a specific product. This article begins by addressing the installation of Facebook's Pixel on a website and theoretically demonstrating its potential to assist digital marketing campaigns. Finally, it discusses a real-world example of using Pixel in a paid ad conversion campaign on Facebook and discusses the results achieved.
\end{abstract}

Keywords: Facebook. Pixel. Digital Marketing. Conversion Campaigns. 


\section{INTRODUÇÃO}

No universo do marketing digital, pode-se afirmar que atualmente o Facebook é um dos principais meios utilizados para se construir e utilizar campanhas de anúncio pago, por se tratar da maior mídia social da atualidade, com 2,3 bilhões de usuários. Assim, acaba sendo um destino muito atrativo para empresas investirem em anúncios (G1, 2019).

Não ao acaso, a tecnologia oferecida pelo Facebook para otimizar anúncios evoluiu e cresceu desde o seu início, assim se tornando uma plataforma mais atrativa para anunciantes. Além de diversas informações importantes para criação de anúncios, como criação de público personalizado, segmentação por idade, interesses e região, conta também com a ferramenta Pixel de rastreamento para campanhas de conversão.

Segundo Faustino (2019), para campanhas de anúncio onde o objetivo é a conversão, ou seja, o objetivo é aumentar ao máximo uma determinada interação desejada por parte dos usuários dentro do site, o Pixel se torna uma ferramenta essencial, pois através dela se consegue mensurar a eficácia de um anúncio através do rastreamento e marcação feito por ele, possibilitando ao anunciante descobrir se deve manter o anúncio ou não.

Pelo fato do Pixel também marcar todos os usuários que tiveram alguma interação com o site, através de seu algoritmo de Inteligência Artificial, o Pixel vai se otimizando automaticamente através de alimentação própria, conforme vai aumentando o número de usuários interagindo com o site, buscando qual é o perfil ideal de clientes para um determinado tipo de anúncio e, assim, possibilitando uma assertividade maior do anúncio aparecer para os usuários provavelmente interessados, além de potencialmente aumentar as chances de melhorar ainda mais o poder do anúncio pago em relação ao seu objetivo de conversão (MARSHALL; KRANCE; MELOCHE, 2017).

\section{INICIANDO COM O PIXEL DO FACEBOOK}

Antes de se analisar a influência do Pixel em anúncios pagos, faz-se necessário discorrer sobre os tipos de anúncio no Facebook e os procedimentos para criação de um anúncio pago de Conversão com a subsequente geração e inserção do código do Pixel no website de destino da campanha, o que é abordado na sequência. 


\subsection{Criação de um anúncio pago no Facebook}

Conforme demonstra o Gerenciador de Anúncios do Facebook Inc. (2019b), existem três categorias principais de campanhas: Reconhecimento, Consideração e Conversão.

Cada uma das categorias principais de campanhas de anúncios pagos possui suas próprias subcategorias e objetivos únicos, os quais o Facebook usará dinheiro investido da melhor forma para conseguir atingir os objetivos e otimizar os custos visando alcançar um maior resultado através da campanha. Ainda segundo Facebook Inc. (2019a), os objetivos gerais de cada uma das categorias principais são:

- Reconhecimento: neste modelo, o objetivo é fazer o anúncio atingir o maior número de usuários dentro da plataforma, baseado em alcance de usuários únicos, independentemente da relação que os usuários tenham com o anúncio.

- Consideração: neste modelo, o objetivo é fazer o anúncio atingir o máximo de interações possíveis no Facebook, como curtidas, compartilhamentos, comentários e tempo de visualizações em vídeos.

- Conversão: neste modelo, o objetivo é proporcionar conversão com o melhor custo-benefício possível, ou seja, mostrar o anúncio para o menor número de pessoas, restringindo ao público segmentado por seu interesse potencial no objetivo do anúncio, ao mesmo tempo em que se almeja aumentar a conversão deste público em ações esperadas no destino da campanha.

Desta forma, é justamente na categoria de campanha de Conversão em que o Pixel de rastreamento se torna necessário, pois as métricas deverão ser extraídas não apenas de dentro da plataforma Facebook, mas sim de um website externo, tornando-se necessário a conexão da plataforma com o site de destino, através do código do Pixel.

Dentro do modelo de campanha de Conversão ainda existem 17 tipos de eventos padrão na qual o Pixel pode fazer o rastreamento, por exemplo: um cadastro no site, uma compra iniciada, um item adicionado a um carrinho de compras, ou mesmo uma compra efetivada através de algum mecanismo de intermediação de pagamento. Além desses eventos padrão, também é possível personalizar eventos a partir das necessidades do anunciante.

Após a escolha do tipo de campanha, considerando que se trata de campanha de Conversão, faz-se necessário gerar o código do Pixel de rastreamento e sua posterior instalação nas páginas onde há o desejo de ser feito o rastreamento. Após estes passos, a 
construção do anúncio propriamente dito se torna comum, como qualquer outra categoria, fornecendo opções para segmentar o público com restrições por interesse, região, idade, além da própria criação do anúncio com sua imagem principal, texto de vendas e um CTA (Call to Action), ou seja, um botão de chamada para ação (FAUSTINO, 2019).

\subsection{Gerando e inserindo o código do Pixel}

Para o Pixel conseguir iniciar o processo de rastreamento, primeiramente deve ser criado, uma vez que cada conta de anúncios deve possuir um código gerado diferente, para que o rastreamento seja viável. Para isso, é necessário ter acesso à uma conta de anúncio na plataforma do Facebook para Empresas (Facebook Business), sendo que a criação do Pixel ocorre dentro de seu Gerenciador de Anúncios.

Conforme é possível observar através da Figura 1, o código do Pixel possui uma estrutura padrão a ser inserida no website a ser rastreado, porém o número em destaque é o código de identificação exclusiva da conta de anúncio (FACEBOOK INC, 2019a).

Figura 1 - Código do Pixel do Facebook

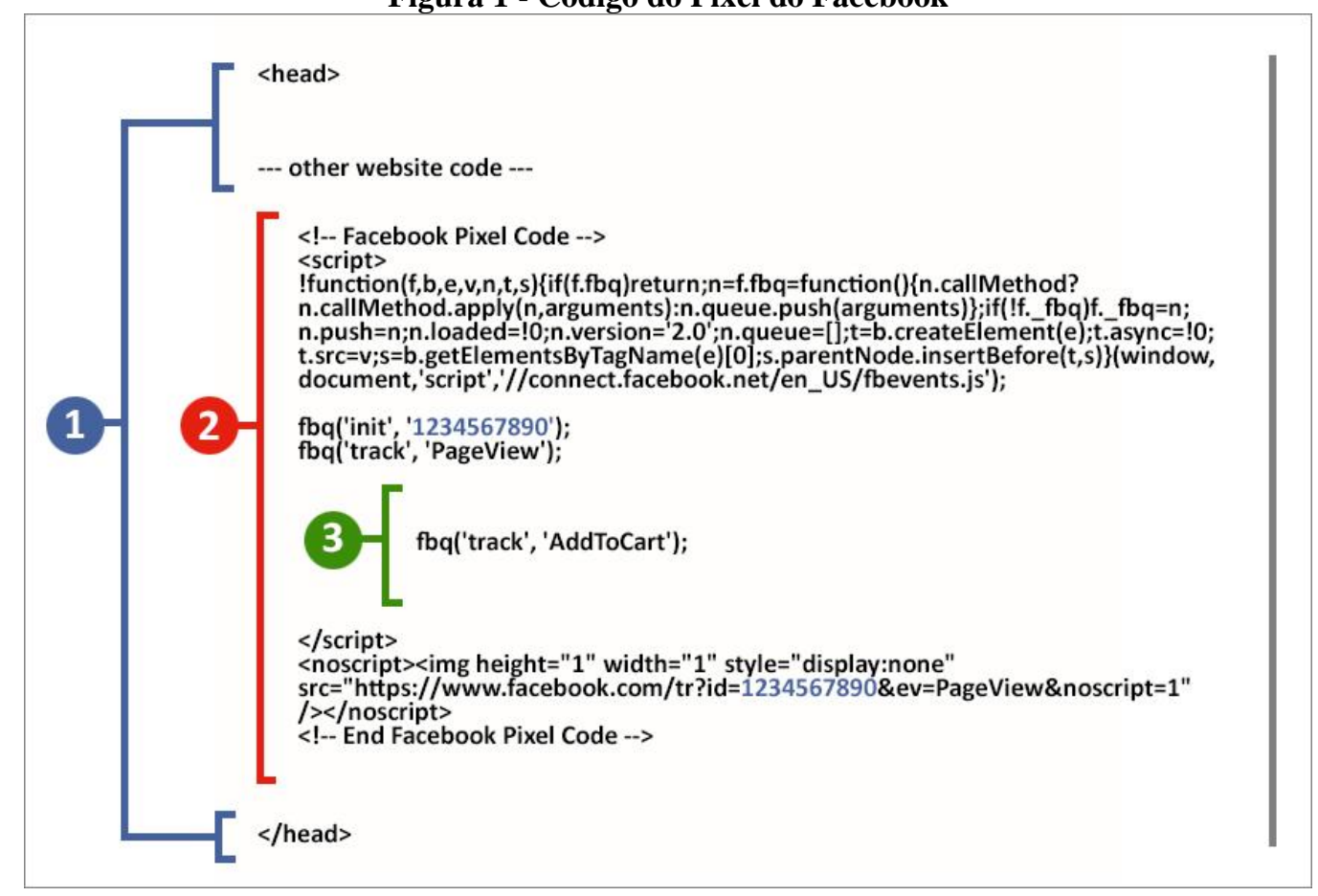

Fonte: Follow Your Arrow Marketing (2016). 
Uma vez que o código é instalado em uma página $W e b$, ressalta-se a importância em compreender que o rastreamento passa a ser realizado independentemente da origem do tráfego, ou seja, ainda que o usuário venha a partir de um local diferente do próprio anúncio no Facebook, o rastreamento ocorrerá. Desta forma, caso o usuário possua uma conta do Facebook salva e autenticada no navegador, ele também será reconhecido pelo Pixel.

\section{POTENCIAL DO PIXEL NOS ANÚNCIOS PAGOS DO FACEBOOK}

A partir do momento em que o Pixel passa a ser utilizado em uma página $W e b$, todos os usuários que acessarem a página e interagirem com ela serão marcados pelo Pixel, possibilitando alguns adicionais para a criação de anúncios no Facebook para Empresas, sendo possível elencar exemplos de potencial, conforme apresentado a seguir.

\subsection{Exemplos de potencial do Pixel}

A partir dos dados rastreados e coletadas através do Pixel do Facebook, passa a ser possível a criação de anúncios com públicos look a like (ou públicos semelhantes), que são públicos gerados pelo próprio Facebook de forma a ser composto por um grupo de usuários que mais se pareçam com os usuários frequentemente visitantes do site, fornecidos ao Facebook pelo Pixel - embora também seja possível montar o público look a like a partir de uma lista de e-mails ou de telefones (COUTINHO, 2018).

Além disso, públicos personalizados também podem ser criados a partir do Pixel, sendo possível englobar apenas pessoas que fizeram algum tipo de interação específica com a página, por exemplo, um cadastro ou uma inicialização de compra, permitindo que o anúncio seja extremamente segmentado para os usuários de maior interesse.

Outra possibilidade oferecida são as campanhas de retargeting ou remarketing, ou seja, a criação de um anúncio específico direcionado apenas às pessoas que, em algum momento, foram marcadas pelo Pixel. Com isso, gera-se uma segunda interação com o usuário que anteriormente interagiu em uma determinada página do site (TIKNO, 2017). 


\subsection{Pixel para campanhas de Conversão}

Campanhas de conversão são a situação onde o Pixel mais se destaca, pois a partir de um objetivo determinado, a ferramenta medirá a interação dos usuários com o website e, através de uma métrica de otimização própria, ela consegue construir automaticamente dados comparativos entre os usuários que interagiram com o objetivo estabelecido, para que futuramente o anúncio possa ser direcionado a usuários com perfis parecidos com os que fizeram a interação desejada. Desta forma, é possível otimizar o investimento financeiro na campanha, potencialmente obtendo-se melhores resultados.

O Pixel do Facebook é ideal para acompanhar todas as atividades realizadas no seu site depois de os utilizadores visualizarem um anúncio seu, por exemplo. É idealmente importante numa estratégia de otimização da performance de anúncios, uma vez que o Facebook passa a entender melhor quem é o seu público e como é que ele compra. Por fim, o Pixel ajuda-o também a encontrar novos potenciais clientes para o seu negócio, com base nas ações realizadas no seu site (FAUSTINO, 2019, p. 118).

No Gerenciador de Anúncios do Facebook, o padrão mínimo para a otimização do Pixel em um anúncio são 50 eventos de conversão dentro de 7 dias, para que o Pixel consiga ter dados o suficiente para conseguir traçar o perfil de usuários com maiores chances de serem convertidos pelo anúncio (FACEBOOK INC, 2019c). Porém, ainda que já atingido o mínimo para a otimização do Pixel, esta não acaba, sendo um processo contínuo e evolutivo, para que o perfil do público cada vez mais seja traçado de forma ideal para o anúncio.

O grande diferencial é que, ainda segundo Facebook Inc. (2019c), durante o período de otimização do Pixel é completamente normal que o desempenho de um anúncio sofra oscilações por conta dos testes que o Pixel faz para que a Inteligência Artificial da plataforma consiga comparar os dados dos usuários que geram eventos através do Pixel, assim conseguindo traçar finalmente o público ideal para o anúncio.

\section{APLICAÇÃO PRÁTICA EM UMA CAMPANHA DE CONVERSÃO}

Com base nas informações previamente apresentadas, foi conduzido um estudo de caso de uma situação real de utilização do Pixel de rastreamento do Facebook, a fim de testar se a otimização do anúncio efetivamente aconteceria conforme esperado, em uma campanha de Conversão criada e configurada com o objetivo "Compra". 
Foi feita uma análise de 7 dias iniciais da campanha antes do Pixel ter sido otimizado, seguida de uma análise de 7 dias durante o processo de otimização do Pixel e, por fim, uma análise de 7 dias após a otimização. Vale enfatizar também que, nesta campanha de Conversão, se utilizou a mesma imagem, o mesmo texto promocional e a mesma segmentação de público, com a única diferença sendo o período de otimização do Pixel.

A principal métrica para comparação entre as campanhas foi o cálculo de Retorno Sobre Investimento Publicitário (ou simplesmente ROAS, de Return on Advertising Spend), uma vez que foram usados orçamentos diferentes e, portanto, essa se demonstrou uma métrica mais adequada para as comparações neste cenário.

\subsection{Cálculo do ROAS}

O ROAS é uma das métricas disponíveis para se avaliar se um anúncio está gerando o resultado esperado, baseado no dinheiro gasto no investimento e em seu respectivo retorno. Por conta disso, o ROAS é uma das métricas mais importantes para se analisar um anúncio, por isso ela é o principal ponto a ser observado (BRITO, 2017).

Conforme este autor, o seu resultado é obtido utilizando o valor de toda a receita retornada com o anúncio, sendo então dividida pelo valor gasto com o próprio anúncio. Alguns exemplos de resultado possíveis para o ROAS:

- Resultado menor que 1 (ou menor que 100\%): significa que o anúncio está dando prejuízo, pois o valor investido é maior que o recebido.

- Resultado igual a 1 (ou igual a 100\%): neste caso, o anúncio não está gerando lucro, porém também não existe a perda de dinheiro.

- Resultado maior que 1 (ou maior que 100\%): significa que o anúncio está gerando lucro, sendo este o valor base para se calcular a quantidade de lucro.

\subsection{Análise de 7 dias iniciais antes da otimização do Pixel}

Conforme previamente abordado, durante o período de otimização do Pixel, o anúncio pode sofrer variações de resultado, inclusive melhorando ou piorando a eficiência dele, por estar em fase de testes, segundo informa Facebook Inc. (2019c). 


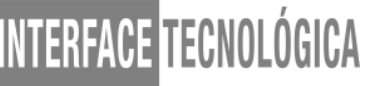

No estudo de caso conduzido, foi possível observar o desempenho da campanha nos 7 primeiros dias, de 8 de julho de 2019 até 14 de julho de 2019, onde o Pixel ainda estava em fase de otimização realizando os testes do Facebook para encontrar o melhor público para o anúncio. Ainda assim, conforme apresenta a Figura 2, já obtendo retorno financeiro.

Figura 2 - Resultado do anúncio nos primeiros 7 dias de veiculação

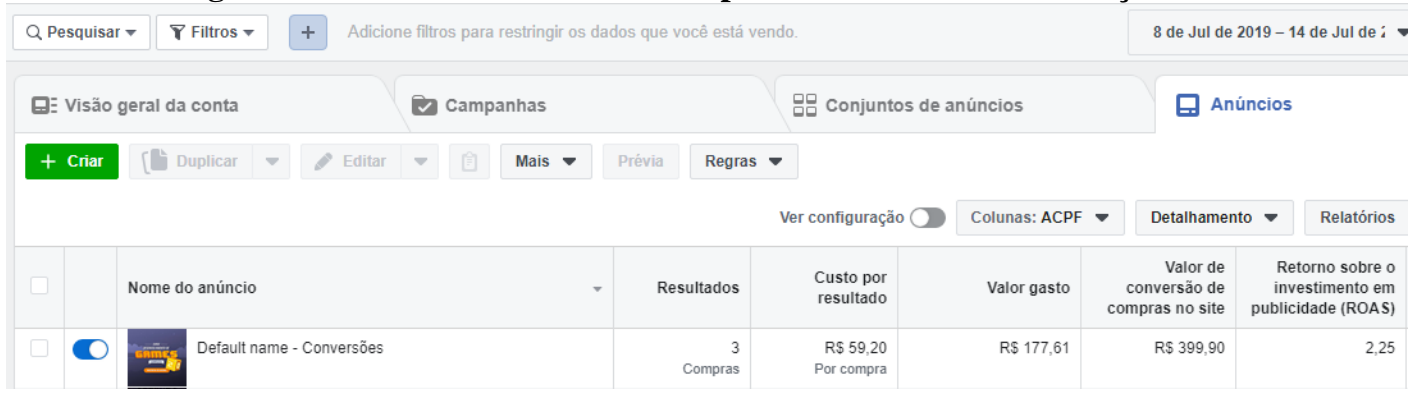

Fonte: Próprio autor (2019).

Ou seja, tal campanha já começou gerando resultados de eventos mesmo em sua primeira semana de veiculação, pois, com um valor gasto de $\mathrm{R} \$ 177,61$, retornou 3 eventos de compra que resultaram em $\mathrm{R} \$ 399,90$, ou seja, um ROAS de 2,25 ou $225 \%$.

É possível supor que, ainda sem ter atingido as metas de otimização do Pixel do Facebook, o anúncio foi bem sucedido em sua combinação de imagem, texto de vendas e público alvo, se demonstrando um bom anúncio desde o princípio.

\subsection{Análise de 7 dias durante a otimização do Pixel}

Uma segunda análise foi realizada durante os 7 dias do período de otimização de resultados do Pixel, entre os dias 18 de julho de 2019 e 24 de julho de 2019. Neste período percebeu-se que, de fato, ocorrem oscilações de resultado. Conforma a Figura 3 demonstra, a campanha que tinha um ROAS de 2,25 passou a gerar 1,20 (ou 120\%) de retorno com relação ao investido financeiramente no anúncio, como pode se observar na imagem abaixo. 


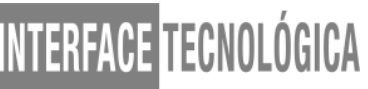

Figura 3 - Resultado do anúncio de 7 dias de veiculação durante a otimização

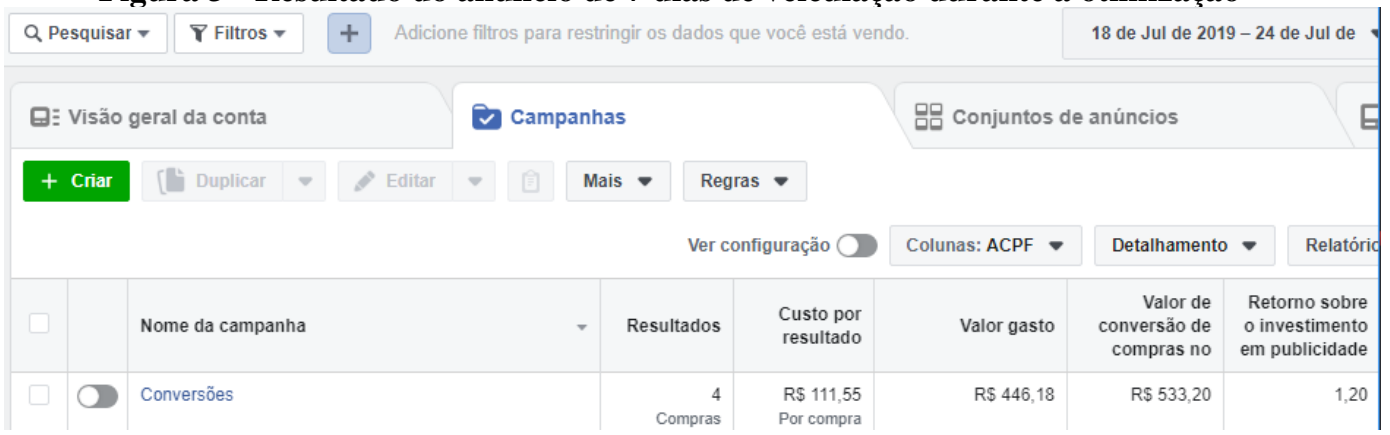

Fonte: Próprio autor (2019).

Visto que este foi período em que o Pixel esteve testando diferentes públicos, as oscilações seriam esperadas. Neste caso específica, atingiu pessoas menos interessadas.

\subsection{Análise de 7 dias após a otimização do Pixel}

$\mathrm{Na}$ imagem abaixo, observa-se o mesmo anúncio, com as mesmas características do início, porém após as metas de otimização do Pixel terem sido concluídas. Naquele momento, no período de 7 dias entre os dias 5 de agosto de 2019 a 11 de agosto de 2019, o Facebook já tinha um padrão definido do público ideal para o anúncio.

Em teoria, este período contaria com informações do melhor público para o anúncio já traçadas. O estudo de caso corroborou esta teoria, conforme a Figura 4 demonstra.

Figura 4 - Resultado do anúncio nos 7 dias de veiculação após a otimização

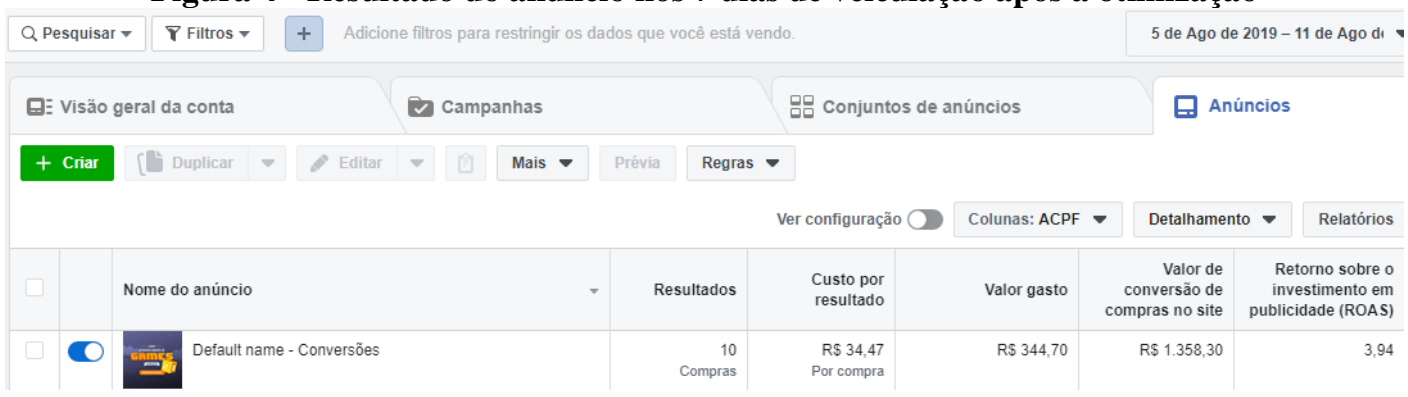

Fonte: Próprio autor (2019).

Apesar de o valor investido no anúncio ter sido quase o dobro dos primeiros sete dias, em virtude de uma expectativa de manutenção de um retorno proporcional ao obtido nos períodos anteriores, ocorreu na verdade um forte aumento no retorno, diminuindo-se o custo por resultado e tornando o anúncio ainda mais lucrativo. 


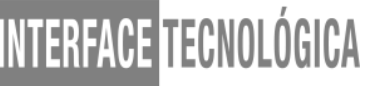

Dessa forma, fazendo com que o mesmo anúncio porém direcionado a um público mais bem selecionado, o Pixel permitiu que se obtivesse quase o dobro do resultado obtido no início da campanha. Com $\mathrm{R} \$ 344,70$ investidos, se obteve $\mathrm{R} \$ 1.358,30$, ou seja, um ROAS de 3,94 (ou 394\%), bem superior ao anterior de 1,20 e ao inicial de 2,25.

Até este momento do estudo, focado somente em uma campanha, os resultados apresentaram grande efetividade do Pixel do Facebook, demonstrando capacidade de otimizar ainda mais uma campanha de Conversão que, desde o início, obteve resultado positivo.

\subsection{Pixel otimiza campanhas apenas com base nos resultados}

Além do caso anteriormente apresentado, outro teste foi conduzido com outra campanha, desta vez com imagem, texto de vendas e público alvo preliminar diferentes.

Além disso, de forma totalmente inversa ao cenário anterior, tal campanha de anúncio teve um início ruim, gerando prejuízo. Ou seja, o Pixel não teria alimentação com os dados de clientes logo no início da campanha, para poder assim otimizar facilmente. Isto potencialmente dificultaria o processo de traçar e alcançar os melhores clientes.

O anúncio foi veiculado com um valor investido de $\mathrm{R} \$ 396,65$ ao longo de 14 dias, compreendidos de 20 de maio de 2019 a 2 de junho de 2019, conforme apresenta a Figura 5.

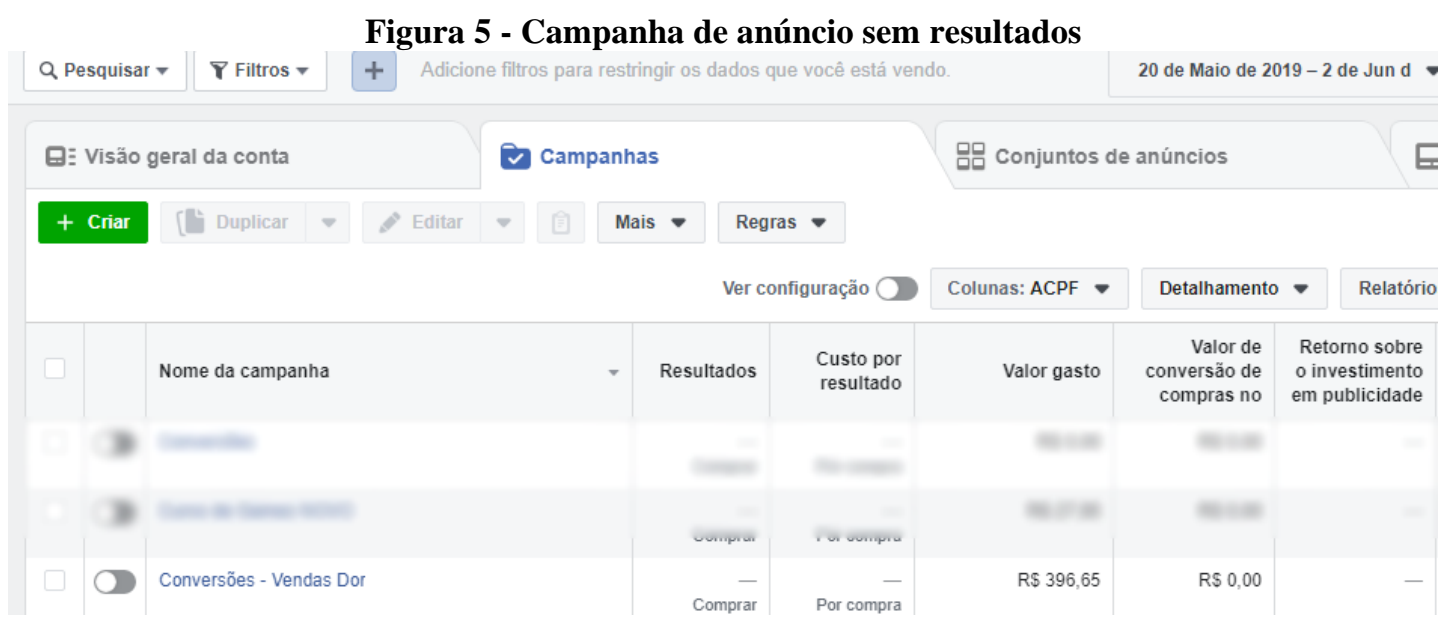

Fonte: Próprio autor (2019).

Assim, a partir dos resultados observados neste anúncio, é possível supor que o Pixel de rastreamento possui realmente potencial para otimizar uma campanha, gerando um resultado maior, desde que seja em um anúncio que já estava gerando resultados. Porém, 


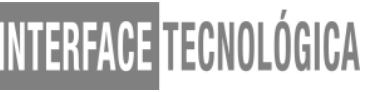

conforme o segundo teste demonstrou, com um anúncio que não estava gerando resultado mesmo após 14 dias e um investimento de $\mathrm{R} \$ 396,55$, o Pixel não foi capaz de ajudar a reverter a situação e a campanha continuou sem gerar resultado algum.

Sendo assim, é possível afirmar que um anúncio mal planejado, sem resultados positivos preliminar, torna o Pixel irrelevante, uma vez que a falta de alimentação de dados de usuários compradores impede o Pixel de alavancar os resultados.

\section{CONCLUSÃO}

O presente trabalho apresentou a ferramenta Pixel do Facebook como mecanismo de rastrear as ações de usuários em um website a vinculado a uma conta de anúncios no Facebook, bem como seu potencial em otimizar campanhas de Conversão na plataforma.

Tanto com base na teoria apresentada sobre o Pixel quanto pelo estudo de caso apresentado, foi possível constatar que o potencial da ferramenta fica evidente em campanhas cujo anúncio já tenha começado a gerar algum resultado positivo, possivelmente otimizando as campanhas e trazendo um resultado maior pelo mesmo custo.

A fundamentação teórica também demonstrou que a utilização do Pixel não é essencial em qualquer tipo de anúncio. Em campanhas onde o objetivo é apenas atrair interação para uma publicação dentro da própria rede social do Facebook, ou mesmo quando o objetivo é alcançar o máximo de pessoas, o Pixel não tem utilidade.

Também a partir do estudo de caso apresentado, ficou caracterizado que a utilização do Pixel não garante ROAS positivo em uma campanha que não tenha obtido resultado algum em seu início de veiculação, uma vez que sem a alimentação de dados de compradores a ferramenta não consegue traçar o perfil do cliente ideal. Desta forma, fica evidente que o mais importante para um bom resultado é um bom anúncio.

Por fim, o presente estudo conclui que o Pixel do Facebook é realmente uma ferramenta poderosa, porém não substitui a ação humana de um bom profissional de marketing digital para gerar resultado através de anúncios pagos.

\section{REFERÊNCIAS}

BRITO, M. ROAS x ROI - Qual a Diferença. MzClick, 2017. Disponível em: <www.mzclick.com.br/roas-X-roi-qual-a-diferenca>. Acesso em: 31 ago. 2019. 


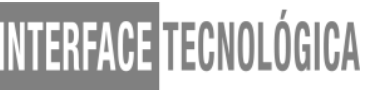

COUTINHO, V. The Social Book: tudo o que precisa de saber sobre o Facebook. Leya, 2018.

FACEBOOK INC. Pixel do Facebook: Sobre o Pixel do Facebook. 2019a. Disponível em: <www.facebook.com/business/help/742478679120153>. Acesso em: 31 ago. 2019.

FACEBOOK INC. Pixel do Facebook: Sobre os Eventos Personalizados e o Padrão do Pixel. 2019b. Disponível em: <www.facebook.com/business/help/964258670337005>. Acesso em: 31 ago. 2019.

FACEBOOK INC. Veiculação de Anúncio: Sobre a Fase de Aprendizado. 2019c. Disponível em: <www.facebook.com/business/help/112167992830700〉. Acesso em: 31 ago. 2019.

FAUSTINO, P. Marketing Digital na Prática: como criar do zero uma estratégia de marketing digital para promover negócios ou produtos. DVS Editora, 2019.

FOLLOW YOUR ARROW MARKETING. The Facebook Pixel: Here's How to Implement It. Follow Your Arrow Marketing Blog, 06 fev. 2016. Disponível em: $<$ followyourarrowmarketing.com/wp-content/uploads/2016/01/Facebook-PixelImplementation.pdf >. Acesso em: 05 set. 2019.

G1. Facebook completa 15 anos com 2,3 milhões de usuários. 2019. Disponível em: <g1.globo.com/economia/tecnologia/noticia/2019/02/04/facebook-completa-15-anos-com-23bilhoes-de-usuarios.ghtml>. Accessor em: 31 ago. 2019.

MARSHALL, P; KRANCE, K; MELOCHE, T. Ultimate Guide to Facebook Advertising: How to Access 1 Billion Potential Customers in 10 Minutes. Entrepreneur Press, 2017.

TIKNO, T. Exploring the Acceptance for Pixel Technology Implementation in Facebook Ads Among Advertisers in Indonesia. KnE Social Sciences, v. 3, n. 1, p. 96-107, 2018. 